\title{
Title: NMR spectroscopy of native and in vitro tissues implicates polyADP ribose in biomineralization
}

\author{
Authors: W. Ying Chow ${ }^{1}$, Rakesh Rajan², Karin H. Muller ${ }^{3}$, David G. Reid ${ }^{1}$, Jeremy N. \\ Skepper ${ }^{3}$, Wai Ching Wong ${ }^{1}$, Roger A. Brooks², Maggie Green ${ }^{4}$, Dominique Bihan ${ }^{5}$, \\ Richard W. Farndale ${ }^{5}$, David A. Slatter ${ }^{5}$, Catherine M. Shanahan ${ }^{6}$, Melinda J. Duer ${ }^{1 *}$
}

\author{
Affiliations: \\ ${ }^{1}$ Department of Chemistry, University of Cambridge, Lensfield Road, Cambridge CB2 \\ $1 \mathrm{EW}, \mathrm{UK}$. \\ ${ }^{2}$ Orthopaedic Research Unit, University of Cambridge, Addenbrooke's Hospital, Cambridge \\ CB2 0QQ, UK. \\ ${ }^{3}$ Department of Physiology, Development \& Neuroscience, University of Cambridge, \\ Downing Site, Cambridge CB2 3DY, UK. \\ ${ }^{4}$ Central Biomedical Resources, University of Cambridge , School of Clinical Medicine, \\ West Forvie Building, Forvie Site, Robinson Way, Cambridge CB2 0SZ, UK. \\ ${ }^{5}$ Department of Biochemistry, University of Cambridge, Downing Site, Cambridge CB2 \\ 1QW, UK. \\ ${ }^{6}$ BHF Centre of Research Excellence, Cardiovascular Division, James Black Centre \\ King's College London, 125 Coldharbour Lane, London SE5 9NU, UK \\ *Correspondence to: mjd13@cam.ac.uk
}

\begin{abstract}
:
NMR spectroscopy is useful to determine molecular structure in tissues grown in vitro only if their fidelity, relative to native tissue, can be established. Here we use multidimensional NMR spectra of animal and in vitro model tissues as fingerprints of their respective molecular structures, allowing us to compare the intact tissues at atomic length scales. To obtain spectra from animal tissues, we developed a heavy mouse enriched by $\sim 20 \%$ in the NMR-active isotopes ${ }^{13} \mathrm{C}$ and ${ }^{15} \mathrm{~N}$. The resulting spectra allowed us to refine an in vitro model of developing bone, and to probe its detailed structure. The identification of an unexpected molecule, poly(ADP ribose), that may be implicated in calcification of the bone matrix, illustrates the analytical power of this approach.
\end{abstract}

One Sentence Summary: Solid-state NMR spectroscopy on ${ }^{13} \mathrm{C},{ }^{15} \mathrm{~N}$-enriched mouse tissue guided development of an in vitro model of developing bone, in which poly(ADP ribose) was unexpectedly found.

\section{Main text:}

Understanding the atomic-level structure of the extracellular matrix (ECM) of tissues is a prerequisite for detailed understanding of both biological and mechanical 
functions of those tissues. However, whilst nanoscopic and longer structural lengthscales can be studied by various forms of microscopy (1-3), there is a paucity of techniques able to probe atomic-level structures in such complex and heterogeneous materials as the ECM. Multidimensional NMR spectroscopy is capable in principle of yielding structural information (4-6), even in such a difficult situation, but only if ${ }^{13} \mathrm{C}$ and ${ }^{15} \mathrm{~N}$ labeling of the molecular components of interest can be achieved. Specific isotope labeling is difficult in vivo, but there are, at least in principle, many more possibilities for such labeling in vitro. The problem is that there is no certainty that an in vitro-grown tissue will have atomic-level structures representative of those in vivo, and worse, there are currently no ways of directly checking the similarity of in vivo and in vitro tissues on this lengthscale. We show here that the structural sensitivity of multidimensional solid-state NMR spectra can solve both of these problems, allowing us to refine as well as validate the atomic structure of an in vitro model of developing bone. Probing the atomic structures and composition of the ECM by NMR requires no a priori knowledge; this feature, in combination with the feasibility of specific isotope labeling in our in vitro model that is not possible in vivo, has allowed us to detect the presence of an unexpected sugar species at the calcification front in developing bone that could also be important in pathological calcification.

Our first step was to develop a heavy mouse in which the protein components of its tissues are ubiquitously enriched in ${ }^{13} \mathrm{C}$ and ${ }^{15} \mathrm{~N}(\sim 20 \%)$, so that we could record reference multi-dimensional NMR spectra of native tissues which act as fingerprints for the underlying molecular structures. ${ }^{13} \mathrm{C}$-enriched collagen has previously been produced through feeding with single isotope-enriched amino acids $\left(1-{ }^{13} \mathrm{C}-\mathrm{Gly},{ }^{15} \mathrm{~N}\right.$ Gly and Pro-2- $\left.d_{2}\right)$ $(7,8)$ and ${ }^{15} \mathrm{~N}$ enrichment of tissues is now common practice for quantitative mass spectroscopy. Here though we require enrichment of many amino acid types and with both ${ }^{13} \mathrm{C}$ and ${ }^{15} \mathrm{~N}$, so our protocol took account of the fact that isotope-labeling may adversely affect metabolism. It resulted in a mouse with significant isotopic enrichment of all collagenous tissues. ${ }^{1}$ These tissues allowed high-quality two-dimensional ${ }^{13} \mathrm{C}-{ }^{13} \mathrm{C}$ and ${ }^{13} \mathrm{C}$ ${ }^{15} \mathrm{~N}$ correlation spectra to be recorded on intact tissues (Fig. 1), without need for further sample preparation. For example, in mouse calvarial bone, $\sim 20 \%$ of amino acid residues incorporated are fully ${ }^{13} \mathrm{C},{ }^{15} \mathrm{~N}$-labeled.

As shown in Figure 1, two different NMR spectral analyses were performed on each tissue sample:

${ }^{13} \mathrm{C}-{ }^{13} \mathrm{C}$ double-quantum - single-quantum (DQ-SQ) spectrum (9) (Fig. 1A) which consists of pairs of ${ }^{13} \mathrm{C}$ signals from pairs of close, i.e. bonded, carbons (within $2 \AA$ ) in the so-called SQ spectral dimension which are centered at the sum of their respective signal frequencies in the DQ dimension. This type of spectrum resolves the detailed ${ }^{13} \mathrm{C}$ chemical shift distributions for each carbon signal; in turn, the chemical shift distribution reflects the distribution of molecular geometries present in the sample.

(ii) ${ }^{13} \mathrm{C}-{ }^{13} \mathrm{C}$ proton-driven spin diffusion (PDSD) spectrum (Fig. 1C) which correlates pairs of ${ }^{13} \mathrm{C}$ spins via through-space dipolar coupling (10) potentially allowing

1 See Materials and Methods in the online Supplementary Materials. 
correlations between more distant (up to $\sim 10 \AA$ ) carbon pairs than the DQ-SQ spectrum.

Two-dimensional ${ }^{13} \mathrm{C}-{ }^{15} \mathrm{~N}$ can also be recorded on these tissues, giving ${ }^{15} \mathrm{~N}$ chemical shift distributions and their correlation with those of neighboring ${ }^{13} \mathrm{C}$ sites (Fig. S1).

The fibril-forming collagens that are the major component of mammalian tissues are trimeric triple-helical proteins consisting of G-X-Y repeats, where $\mathrm{X}$ is often proline (Pro, $\mathrm{P}$ ) and $\mathrm{Y}$ hydroxyproline (Hyp, O), a collagen-specific post-translational modification. Collagen signals, in particular those from Gly, Pro and Hyp, dominate all our heavy mouse tissue correlation spectra.

The DQ-SQ spectrum (Fig. 1A) allows resolution of signals that were previously overlapped in one-dimensional spectra of pure collagen (11), enabling measurement of separate ${ }^{13} \mathrm{C}$ signals for Pro C $\alpha$ (59.1 ppm) and Hyp C $\alpha$ (57.8 ppm). The DQ-SQ spectrum further allows resolution and assignment of ${ }^{13} \mathrm{C}$ signals for almost all amino acid types in collagen (see Fig. S1 (C) and Table S1). It is to be noted that the ${ }^{13} \mathrm{C}$ chemical shifts of all the main chain carbons are significantly lower than generally found for the same residues in other proteins; these low chemical shifts are diagnostic of the collagen triple helical motif and occur because the ${ }^{13} \mathrm{C}$ chemical shift is highly sensitive to local molecular geometry, i.e. to peptide dihedral angles (5) and chemical bonding as well as primary sequence (12). As well as resolving the full ${ }^{13} \mathrm{C}$ chemical shift distribution for each amino acid residue type, the DQ-SQ spectrum displays the correlations between the chemical shift distributions of neighboring carbon atoms, and so serves as a sensitive monitor of the detailed local environments for each residue type in the collagen structure. For instance, the glycine $\mathrm{C}_{\alpha}$ and $\mathrm{C}^{\prime}$ signals in the DQ-SQ spectrum each show a distribution of chemical shifts and these are linearly correlated with each other as indicated in Fig. 1A; each pair of correlated $\mathrm{C}_{\alpha}$ and $\mathrm{C}^{\prime}$ Gly chemical shifts within this distribution represents a particular molecular Gly microenvironment, the relative intensity of the correlation indicating the relative abundance of that molecular microenvironment in the overall protein structure.

These two-dimensional NMR spectra thus represent "fingerprints" of the underlying atomic structures within the native mouse tissue. Our second step is to use these fingerprints to determine the fidelity of an in vitro bone model. In vitro tissue was grown from low passage fetal sheep primary osteoblasts in medium containing $\mathrm{U}_{-}{ }^{13} \mathrm{C},{ }^{15} \mathrm{~N}$ labeled glycine and proline ${ }^{2}$, and 2D NMR correlation spectra recorded as above. Comparison of these spectra with those from the heavy mouse bone tissue allowed an immediate assessment of the similarity of the atomic structures between the in vitro and in vivo tissues. Detailed comparison indicated in what respects the two tissues differed on the atomic lengthscale and so suggested ways of improving the cell culture protocol generating the in vitro tissue to produce more native-like tissue. The main spectral differences are some signal intensity to the high frequency side of the Pro, Hyp and Gly signals for the in vitro tissue which are lacking for native tissue (Fig. S2A). The higher frequency of these

2 This results in the hydroxyprolines (post-translationally modified from prolines) also being ${ }^{13} \mathrm{C}$ and ${ }^{15} \mathrm{~N}$ enriched, so that more than $50 \%$ of the total amino acid residues in the collagens are labeled. 
signals indicates that they are from residues not in a collagen triple helical motif. The signals are more intense after the sample has been sonicated (Fig. S2B) and therefore most likely arise from Pro, Hyp and Gly in un/mis-folded collagen-like proteins or fragments, which is consistent with their higher chemical shift values. These signals were reduced by minimizing the shear stress on the cells in culture while they were laying down ECM, by reducing the frequency of culture medium renewal and minimizing disturbance of the cells during this process (Figs. S2C and D). The in vitro tissue was judged to be optimal when the 2D spectra showed a high degree of similarity to those of the native mouse tissue (Figs. $1 \mathrm{~B}$ and $\mathrm{D})$, indicating that the molecular geometries in the two tissues are very similar. Furthermore, SEM of the optimized in vitro ECM (to examine nanoscale structure) showed a highly organized matrix of parallel fibrils, similar to native bone (Fig. S3A and B), and AFM showed correct assembly of triple helices into the characteristic $67 \mathrm{~nm}$ banded fibril (Fig S3C), and based on these results would also be judged as representative of native tissue at a nanoscopic level. There is still some signal from unfolded or mis-folded collagen proteins in the optimized tissue (Fig. 2). Such species may cause deleterious immune responses in tissue grown in vitro for implants, for instance, so knowledge of their presence is important. These species cannot be detected by microscopy without a suitable immune label and, by implication, a priori knowledge of these species, nor would such proteins be expected to affect the collagen fibril structure, thus they would not be apparent under SEM or AFM.

Our refined in vitro tissue model allows us to study the sugar components of a tissue as well as the collagen proteins. The sugars in matrix glycoproteins and proteoglycans are essential parts of the ECM structure - collagen glycosylation for instance is necessary for proper fibril formation-yet atomic structures of sugars in intact tissue are understudied in comparison to proteins, largely because of a lack of methods to probe them. Moreover, we have previously shown that a sugar species yet to be identified is important in the organicinorganic interface in bone (13), so we decided to monitor the sugar species present in developing bone and examine how far collagen glycosylation might be involved in the organic-inorganic interface. Isotope enriching tissue sugars in an animal model would be extremely challenging as sugars are rapidly metabolized but is feasible in our in vitro model. Thus, our next step was to generate ${ }^{13} \mathrm{C}$ enrichment of the sugar species in our in vitro ECM, achieved by addition of $\mathrm{U}_{-}{ }^{13} \mathrm{C}$-glucose to the cell culture medium. This procedure resulted in extensive ${ }^{13} \mathrm{C}$-enrichment of collagen glycosylations (14), which were assigned using typical chemical shifts for the expected glycosylation products of collagen type I supported by information from spectral through-space correlations (Fig. 3A) plus the spectra in Figs. S4-S8, as detailed in Tables S3 and S4. The molecular species previously identified at the organic-inorganic interface in bone have signals in the range $72-78 \mathrm{ppm}$ (and includes a component from mineral-associated citrate at $\sim 76 \mathrm{ppm}$ ). Moreover, it has been shown that all of these signals are due to carbons closer in space to phosphorus (and by implication, mineral phosphate) than collagen protein carbons (Fig. S9) $(13,15)$. The spectrum in Fig. 3A shows that the main signal intensity from the collagen glycosylation here occurs between $72-75 \mathrm{ppm}$ and thus we conclude that collagen glycosylation species 
are predominantly closer to mineral crystals in bone than is the collagen protein itself. Since the mineral crystals in bone are close packed between the collagen fibrils (16), this implies that the collagen glycosylation must lie between the collagen fibrils and mineral crystals, i.e. that the collagen glycosylation must lie predominantly on the collagen fibril surfaces. However, there is further signal intensity due to carbons close in space to phosphorus in bone, in particular a weaker signal at $\sim 66 \mathrm{ppm}$ as shown in Fig. S9 and elsewhere $(13,15)$. There is a broad signal around $66 \mathrm{ppm}$ in our 2D correlation spectra but it does not correlate with any signals from collagen glycosylation carbons; furthermore, we noted that there were other signals in the sugar region of the ${ }^{13} \mathrm{C} 2 \mathrm{D}$ correlation spectra (Fig. 3A) not assignable to either collagen glycosylation nor non-enzymatic protein glycation, nor to typical tissue proteoglycans. Detailed analysis of the signal frequencies and their correlations in two dimensional ${ }^{13} \mathrm{C}-{ }^{13} \mathrm{C}$ PDSD and DQ-SQ correlation spectra (Fig. 3B) show that these correspond closely to the signals that might be expected for PAR and ADP-ribose (ADPR) (assignment in Tables S6 \& S7, Figs. S10, S11). If these signals are indeed from such species, then they must be in an extracellular environment, as all cells have been removed from the in vitro ECMs prior to the NMR experiments (verified by SEM). Furthermore they must be immobilized in the ECM to give signals in the correlation spectra and sufficiently well bound not to be removed by repeated washing during sample preparation.

PAR is a post-translational modification of cellular, particularly nuclear, proteins, and is involved in DNA repair and the regulation of cell survival and death. Neither PAR nor ADPR have been previously reported as components of the ECM, nor to the best of our knowledge has there been any suggestion that they might exist in or form persistent attachments to the ECM. The largely nuclear role documented to date for these species meant that their presence in our in vitro tissue was unexpected. Accordingly, we examined an ex vivo model of developing bone, fetal sheep growth plate, for the presence of PAR by staining and confocal laser fluorescence microscopy (Fig. 4). The images in Fig. 4 show the presence of significant quantities of PAR in the calcifying region of the growth plate in comparison to the adjoining unmineralized hypertrophic cartilage. The PAR appears both in close proximity to cell nuclei, i.e. intracellular, and in regions where there are no cell nuclei, i.e. presumably extracellular. Thus, its presence in our in vitro tissue models has been confirmed in a comparable native tissue model.

PAR is generated in cells from $\mathrm{NAD}^{+}$by PAR polymerase (PARP) enzymes (17, 18). Over-activation of PARPs leads to cellular ATP and $\mathrm{NAD}^{+}$depletion and drives cell necrosis $(17,19)$. Osteoblast necrosis is an essential part of bone mineralization in vivo (20), with $65-85 \%$ of bone osteoblasts suffering this fate within mineralizing zones (21). Osteoblast necrosis is thought to be necessary to make space for the forming mineral crystals but our results here suggest it may have an additional purpose. Cell necrosis results in release of cell contents into the surrounding ECM, and this process is presumably how PAR ends up in the ECM in developing bone. That PAR binds to the ECM at the same time as the matrix is beginning to mineralize is potentially significant. The charged pyrophosphate groups of PAR have high calcium affinity and its polymeric structure makes 
it a candidate as a scaffold for mineral formation. Moreover, PAR has a binding affinity for collagen type XII (22), a FACIT collagen that decorates the surface of collagen type I fibrils, the majority collagen type in bone; as it is between such fibrils that the bulk of bone mineral forms, we conclude that bound PAR can also be in the right place to act as a scaffold for mineral formation. Furthermore, previous work has shown that if organic phosphate esters are removed from demineralized bone, the bone cannot be remineralized (23), suggesting that the species responsible for nucleating bone mineral crystals has been removed by this process. The organic phosphate esters concerned have been assumed to be phosphorylated proteins, but our work shows that there is another possible species, namely the PAR polysaccharide. Other work has shown that polyanions such as poly-aspartate can induce biomimetic mineralization of collagen matrices by stabilizing a mineral precursor phase $(24,25)$, but no such polyanion has yet been reported in the context of native bone ECM. PAR however fits the description. Moreover, we have previously shown that the same distribution of sugar species at the organic-inorganic interface in bone occurs in pathological calcification in arteries as well (26), and cell death/ necrosis always precedes these pathologies (27), so PAR may be a feature in pathological calcification also.

In conclusion, NMR comparisons of ${ }^{13} \mathrm{C},{ }^{15} \mathrm{~N}$-enriched in vivo and in vitro tissues allows the fidelity of the atomic structure of the in vitro model to be ascertained. Our first application of a dual in vivo-in vitro NMR-based approach towards understanding the atomic structures of tissues has led to the identification of a molecule in the ECM of developing bone likely to be relevant to matrix calcification and capable of binding mineral to the collagen matrix. This molecule, PAR, is more commonly associated with nuclear proteins, but most likely to be present in the ECM of forming bone due to cell necrosis associated with bone development. PAR may also be relevant to the initiation of pathological vascular calcification, which we have previously shown involves similar sugar species to those in bone (26). In addition, having the ability to understand differences between an in vitro and in vivo tissue in terms of their respective molecular structureshere the presence of mis-folded collagen proteins in the in vitro tissue - raises the possibility of more rational refinement of in vitro tissue models. 


\section{References and Notes:}

1. S. Weiner, H. D. Wagner, The Material Bone: Structure-Mechanical Function Relations. Annu. Rev. Mater. Sci. 28, 271-298 (1998).

2. S. Bertazzo, T. von Erlach, S. Goldoni, P. L. Çandarlığlu, M. M. Stevens, Correlative Light-Ion Microscopy for biological applications. Nanoscale 4, 2851-4 (2012).

3. W. Denk, H. Horstmann, Serial block-face scanning electron microscopy to reconstruct three-dimensional tissue nanostructure. PLoS Biol. 2, e329 (2004).

4. D. S. Wishart, B. Sykes, The ${ }^{13} \mathrm{C}$ Chemical-Shift Index: A simple method for the identification of protein secondary structure using $13 \mathrm{C}$ chemical-shift data. $J$. Biomol. NMR 4, 171-180 (1994).

5. S. Spera, A. Bax, Empirical correlation between protein backbone conformation and $\mathrm{C} \alpha$ and C $\beta$ 13C nuclear magnetic resonance chemical shifts. J. Am. Chem. Soc. 113, 5490-5492 (1991).

6. A. Böckmann, Structural and dynamic studies of proteins by high-resolution solid-state NMR. Comptes Rendus Chim. 9, 381-392 (2006).

7. S. K. Sarkar, C. E. Sullivan, D. A. Torchia, Solid State ${ }^{13}$ C NMR study of collagen molecular dynamics in hard and soft tissues. J. Biol. Chem. 258, 9762 - 9767 (1983).

8. D. A. Torchia, Y. Hiyama, S. K. Sarkar, C. E. Sullivan, P. E. Young, Multinuclear magnetic resonance studies of collagen molecular structure and dynamics. Biopolymers 24, 65-75 (1985).

9. M. Hohwy, H. J. Jakobsen, M. Edén, M. H. Levitt, N. C. Nielsen, Broadband dipolar recoupling in the nuclear magnetic resonance of rotating solids: A compensated $\mathrm{C} 7$ pulse sequence. J. Chem. Phys. 108, 2686-2694 (1998).

10. N. M. Szeverenyi, M. J. Sullivan, G. E. Maciel, Observation of spin exchange by twodimensional fourier transform $13 \mathrm{C}$ cross polarization-magic-angle spinning. $J$. Magn. Reson. 47, 462-475 (1982).

11. A. E. Aliev, Solid-state NMR studies of collagen-based parchments and gelatin. Biopolymers 77, 230-45 (2005).

12. D. S. Wishart, C. G. Bigam, A. Holm, R. S. Hodges, B. D. Sykes, (1)H, (13)C and (15)N random coil NMR chemical shifts of the common amino acids I Investigations of nearest-neighbor effects. J. Biomol. NMR 5, 332 (1995).

13. E. R. Wise et al., The Organic-Mineral Interface in Bone Is Predominantly Polysaccharide. Chem. Mater. 19, 5055-5057 (2007).

14. R. G. Spiro, Characterization and quantitative determination of the hydroxylysinelinked carbohydrate units of several collagens. J. Biol. Chem. 244, 602-612 (1969). 
15. O. Nikel et al., Solid state NMR investigation of intact human bone quality: balancing issues and insight into the structure at the organic-mineral interface. J. Phys. Chem. C. Nanomater. Interfaces 116, 6320-6331 (2012).

16. E. A. McNally, H. P. Schwarcz, G. A. Botton, A. L. Arsenault, A model for the ultrastructure of bone based on electron microscopy of ion-milled sections. PLoS One 7, e29258 (2012).

17. P. Jagtap, C. Szabó, Poly(ADP-ribose) polymerase and the therapeutic effects of its inhibitors. Nat. Rev. Drug Discov. 4, 421-40 (2005).

18. A. Hakmé, H.-K. Wong, F. Dantzer, V. Schreiber, The expanding field of poly(ADPribosyl)ation reactions "Protein Modifications: Beyond the Usual Suspects" Review Series. EMBO Rep. 9, 1094-100 (2008).

19. H. C. Ha, S. H. Snyder, Poly(ADP-ribose) polymerase is a mediator of necrotic cell death by ATP depletion. Proc. Natl. Acad. Sci. U. S. A. 96, 13978-82 (1999).

20. B. Zimmermann, Occurrence of osteoblast necroses during ossification of long bone cortices in mouse fetuses. Cell Tissue Res. 275, 345-353 (1994).

21. S. C. Manolagas, Birth and death of bone cells: basic regulatory mechanisms and implications for the pathogenesis and treatment of osteoporosis. Endocr. Rev. 21, 115-37 (2000).

22. J.-P. Gagné et al., Quantitative proteomics profiling of the poly(ADP-ribose)-related response to genotoxic stress. Nucleic Acids Res. 40, 7788-805 (2012).

23. M. J. Glimcher, Mechanism of calcification: Role of collagen fibrils and collagenphosphoprotein complexes in vitro and in vivo. Anat. Rec. 224, 139-153 (1989).

24. L. B. Gower, Biomimetic Model Systems for Investigating the Amorphous Precursor Pathway and Its Role in Biomineralization. Chem. Rev. 108, 4551-4627 (2008).

25. A. Dey et al., The role of prenucleation clusters in surface-induced calcium phosphate crystallization. Nat. Mater. 9, 1010-4 (2010).

26. M. J. Duer et al., Mineral surface in calcified plaque is like that of bone: further evidence for regulated mineralization. Arterioscler. Thromb. Vasc. Biol. 28, 2030-4 (2008).

27. F. Otsuka, K. Sakakura, K. Yahagi, M. Joner, R. Virmani, Has Our Understanding of Calcification in Human Coronary Atherosclerosis Progressed? Arterioscler. Thromb. Vasc. Biol. (2014), doi:10.1161/ATVBAHA.113.302642. 


\section{Acknowledgments:}

Advice from Martin Norman and Jo Keeley with technical support from Sally Thomas and Rebecca Westwood (Central Biological Services, University of Cambridge), and helpful interactions with Dominique Martel (SAFE, Augy, France).

Advice and assistance with AFM from Drs Rachel Oliver and Chloe Grover (University of Cambridge).

The authors would like to acknowledge funding from BBSRC for DAS, DGR (BB/G021392/1), MJD, RR \& RWF; BHF for DAS \& RWF (PG/08/011/24416) and KHM (PG/10/43/28390), MJD, JNS, CMS; EPSRC for WYC and WCW; MRC for DB \& RWF (G0500707); National Institute of Health Research for RAB. 
(A)
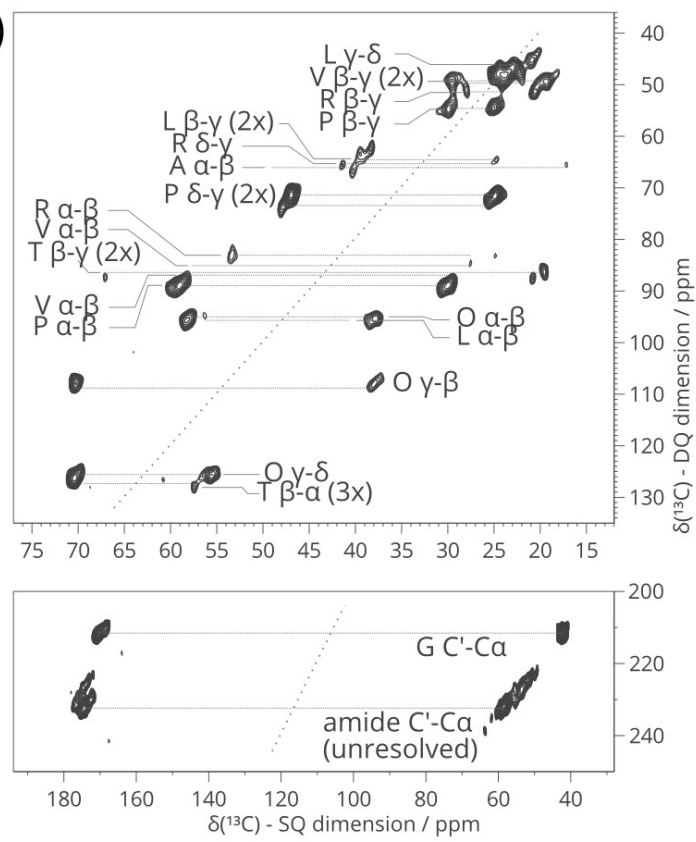

(C)

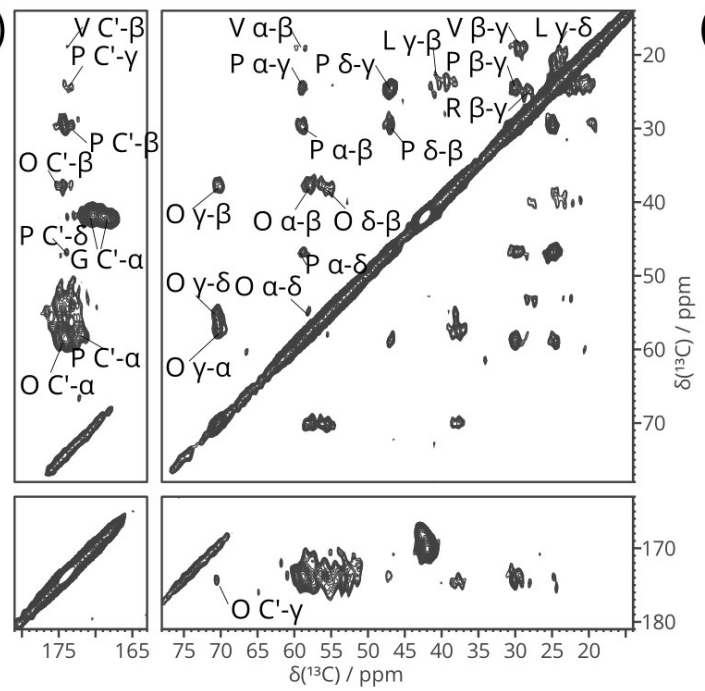

(B)
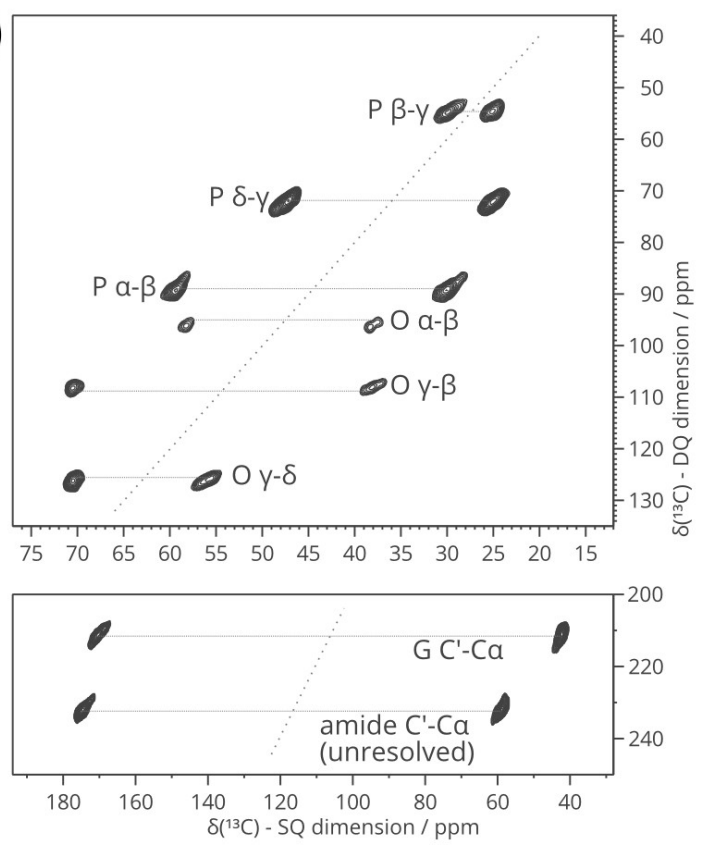

(D)

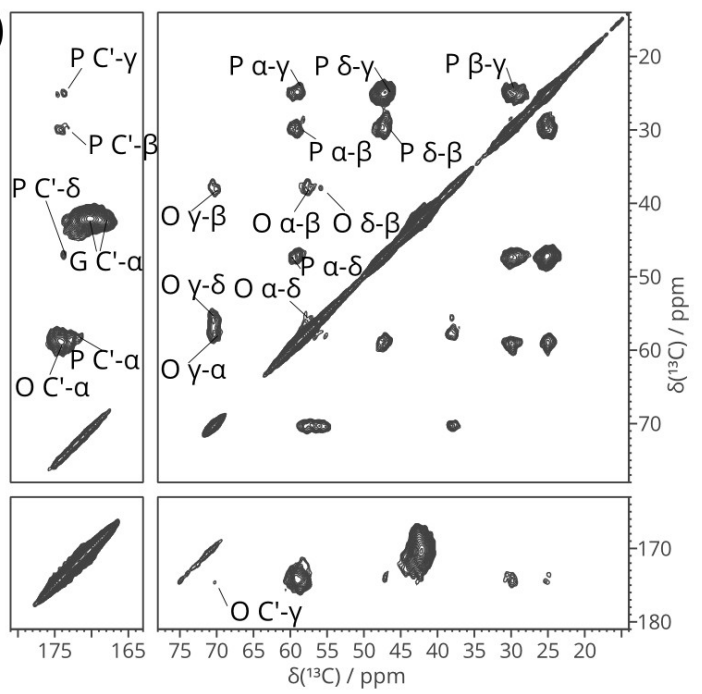


Fig. 1. 2D ssNMR spectra of labeled mouse bone and in vitro ECM. ${ }^{13} \mathrm{C}-{ }^{13} \mathrm{C}$ SQ-DQ correlation spectra from mouse calvarial bone (A) and in vitro fetal ovine osteoblast ECM cultured in the presence of $\mathrm{U}^{13} \mathrm{C}$ Gly, Pro (B). The dotted line indicates the doublequantum diagonal. ${ }^{13} \mathrm{C}-{ }^{13} \mathrm{C}$ PDSD correlation spectra from bone $(\mathrm{C})$ and osteoblast ECM as in (B) (spinning rate, $10 \mathrm{kHz}$, mixing time $20 \mathrm{~ms}$ ) (D). These spectra are symmetric about the diagonal, and hence only half the observed signals are labeled. Other signals are not visible at the current displayed contour level, but shown in Fig. S1 and listed and assigned in Table S1. 


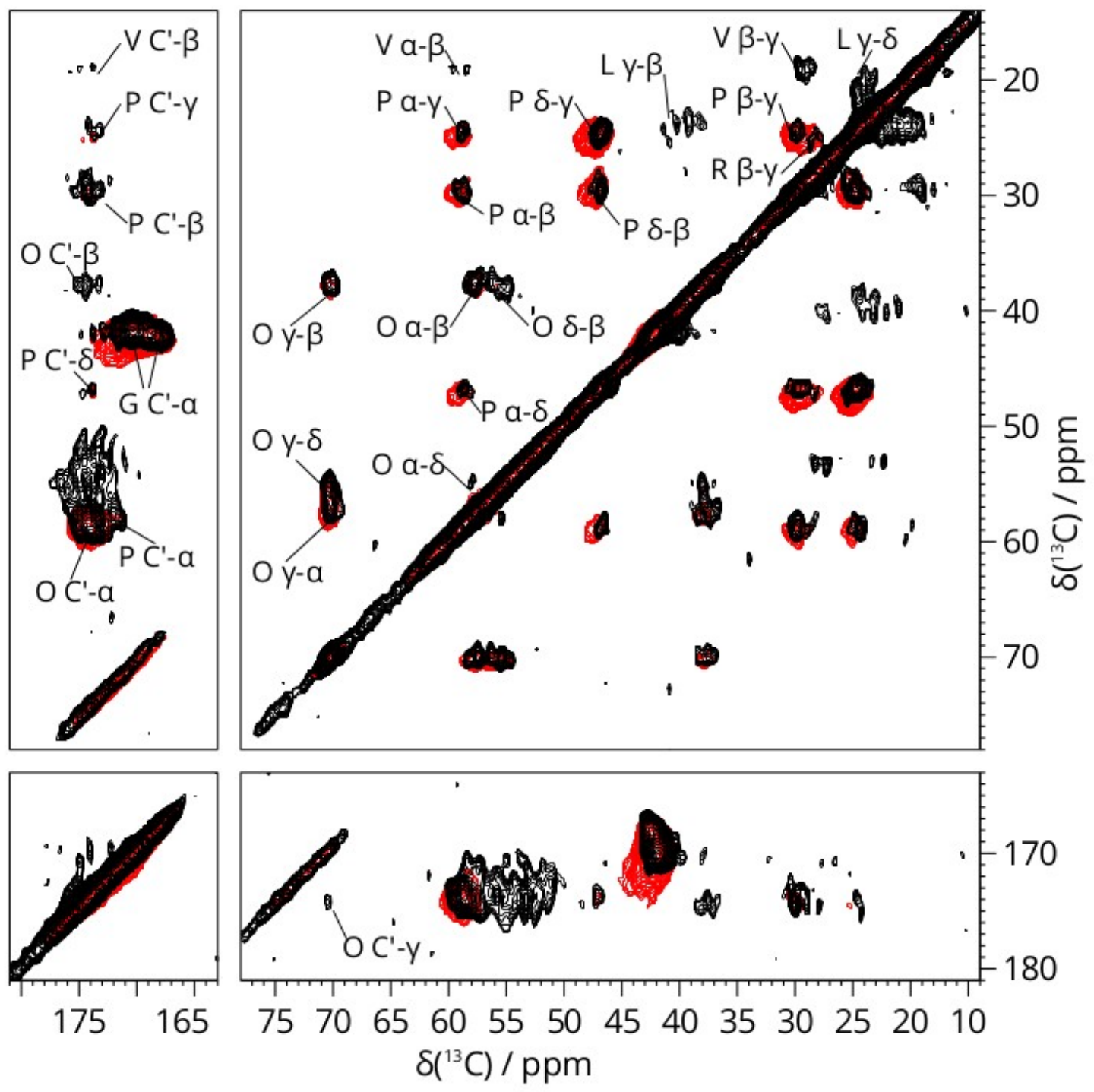


Fig. 2. Overlay of ssNMR PDSD spectra for in vivo and in vitro tissues, highlighting spectral regions where they differ. PDSD spectra of labeled mouse calvarial bone (black) and in vitro fetal ovine osteoblast ECM (red) cultured in the presence of U- ${ }^{13} \mathrm{C}$ Gly, Pro. Differences between the spectra in the range of chemical shift values shown in the red spectrum are likely to arise from un/mis-folded proteins in the in vitro sample (see also Fig. S2 (A)). 


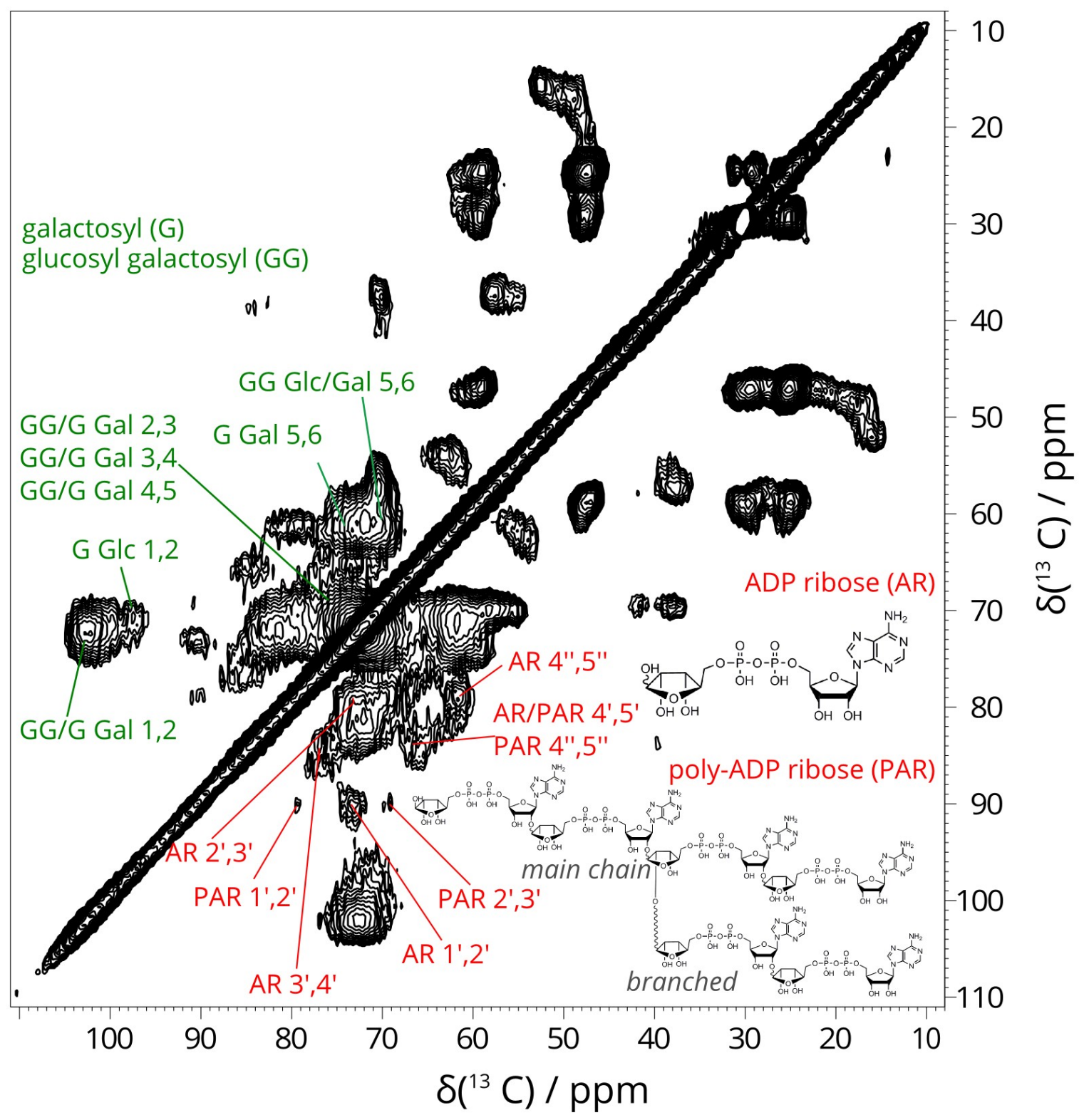


Fig. 3: Identifying poly(ADP ribose) (PAR) in our in vitro model of developing bone. ${ }^{13} \mathrm{C}-{ }^{13} \mathrm{C}$ PDSD correlation spectrum of in vitro fetal sheep osteoblast ECM grown in the presence of $\mathrm{U}^{13} \mathrm{C}$-glucose, Gly and Pro. Signals assigned: O-linked galactosyl (G), Olinked glucosyl galactosyl (GG), both native collagen glycosylation species (green labels, structures not shown). The remaining signals are assigned to ribosyl phosphate species (red labels). Inset: The structures of ADP ribose (AR) and poly(ADP ribose) (PAR). 

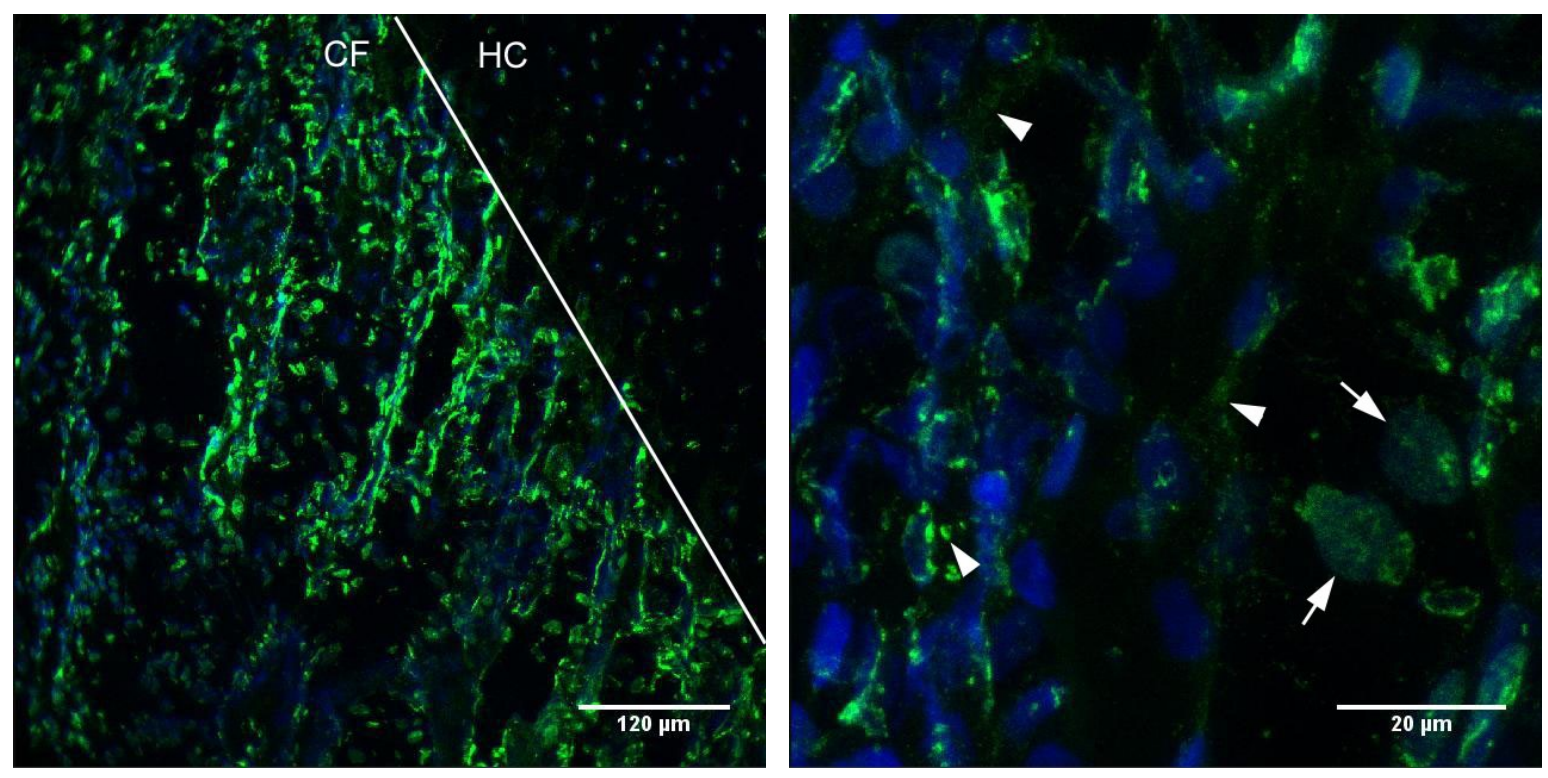

Fig 4: Immunostaining of fetal sheep growth plate shows the presence of PAR.

Maximum intensity projection of Z-stacks. Frozen tissue sections of fetal sheep bone growth plate were stained for nuclei (Hoechst staining, blue) and PAR (clone 10H anti-PAR antibody, green). In the left image, the white line marks the boundary between the zone of hypertrophic cartilage (HC) and the calcification front (CF). Strong PAR staining is visible in the $\mathrm{CF}$, whereas the adjoining zone of $\mathrm{HC}$ is stained weakly. In the right image, in the $\mathrm{CF}$ some PAR staining is co-localized with Hoechst in the nuclei (arrowheads). In addition, PAR staining is also found in the cytoplasm and probably the extracellular space (arrows). 


\section{Supplementary Materials:}

Materials and Methods

Figures S1-S10

Tables S1-S6

References (26-50) 